\title{
Analysis of optimal strategies for soft landing on the Moon from lunar parking orbits
}

\author{
R V RAMANAN and MADAN LAL \\ Aerospace Flight Dynamics Group, Vikram Sarabhai Space Centre, Thiruvananthapuram 695 022, India. \\ *e-mail: rv_ramanan@vssc.org
}

Optimal trajectory design of a probe for soft landing on the Moon from a lunar parking orbit by minimizing the fuel required is obtained. The problem is formulated as an optimal control problem with the thrust direction being the control variable. Using the maximum principle of Pontryagin, the control variable is expressed as a function of co-state variables and the problem is converted into a two-point boundary value problem. The two-point boundary value problem is solved using an optimization technique, i.e., controlled random search. The strategies such as

- direct landing from a lunar parking orbit using powered braking

- direct landing from an intermediate orbit using powered braking

- by executing powered braking in two phases: through horizontal braking and vertical landing

are analyzed and an optimal strategy that achieves the goals is suggested. Also, appropriate design parameters are selected using this analysis.

\section{Introduction}

With the revival of interest in the scientific exploration of the Moon, several new missions are under study by various space agencies. To unravel the topological and morphological mysteries of the Moon, lander missions (Kawakatsu et al 1998; Oono et al 1998) are also being considered. In order to achieve the goals of a lander mission, it is necessary to ensure soft landing of the probe, which generally requires the vertical touch down velocity to be around $5 \mathrm{~m} / \mathrm{s}$ to ensure the safety of the scientific instruments.

Conventionally, landing is initiated from a lunar parking orbit. Since the Moon does not have any appreciable atmosphere, the orbital velocity must be neutralized by appropriate motors/thrusters, a process known as powered braking. To maximize the probe mass, it is essential that the velocity reduction is fuel-optimal. The direction of the thrust must be appropriately varied to ensure soft landing with minimum fuel expenditure. These fuel optimal soft landing trajectories are obtained by formulating the problem as an optimal control problem with the thrust direction angle as the control variable. Vasile and Flobergghagen (1998) followed a temporal finite element method to solve the optimal control problem. In this paper, the optimal profile of the thrust direction is obtained by converting it into a two-point boundary value problem using Pontryagin's maximum principle (Kirk 1970). The solution of the twopoint boundary value problem is obtained using an optimization technique known as controlled random search (Price 1983; Subba Rao and Ramanan 1992). The approach for the solution is discussed in detail.

In addition to the touch down velocity requirement for soft landing, it is essential that the craft lands vertically on the surface of the Moon. Different strategies are explored to identify a suitable strategy that achieves such mission goals. The soft landing can be achieved by powered breaking through many strategies:

Keywords. Lunar parking orbit; soft landing; Pontryagin's maximum principle; two-point boundary value problem; controlled random search. 
- by initiating the powered braking from the lunar parking orbit itself and directly landing,

- by initiating powered braking from an intermediate orbit and directly landing and

- by splitting the powered braking into two phases,

(i) powered horizontal braking phase that ends at a low altitude with a small vertical velocity and

(ii) a vertical descent phase ensuring the required touch down velocity.

The merits and demerits of these strategies are discussed. Some design parameters such as

- initial thrust to mass ratio,

- initial intermediate orbit size from which horizontal braking phase starts and

- altitude and velocity at the end of powered braking, etc., play a critical role in achieving fueloptimal trajectories.

They must be appropriately chosen to achieve the mission goals. This paper addresses these issues and suggests an optimal strategy with appropriate design parameters for a typical soft landing.

\section{Solution process}

\subsection{Problem description}

A space probe in an orbit around the Moon is to be landed on the Moon with near-zero velocity minimizing the fuel expenditure, viz., in minimum time.

\subsection{Assumptions}

- Target landing location is assumed to be in the orbital plane.

- Gravity field of the Moon is considered as spherical.

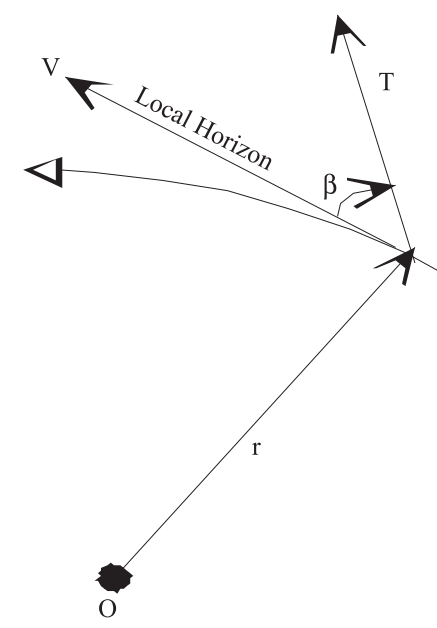

Figure 1. Lander probe under thrusting.
The spherical gravity field is adequate because flight time is about 1000 seconds only and the effect of non-spherical gravity field is negligible. Further, the non-spherical field, if included, the simplicity of the solution procedure will be lost.

\subsection{Governing equations}

The planar motion of the lander probe is represented by the variables, $r, \varphi, u, v$ where $r$ is the radial distance from the center of the Moon; $\varphi$ is the range angle or true anomaly; $u$ is the horizontal velocity; and $v$ is the vertical velocity. The equations describing the motion of the probe under the gravity of the Moon and the acceleration due to the thrust are

$$
\begin{aligned}
\dot{r} & =v, \\
\dot{\phi} & =\frac{u}{r}, \\
\dot{u} & =-\frac{u v}{r}+\frac{T}{M} \cos \beta, \\
\dot{v} & =\frac{u^{2}}{r}-\frac{\mu}{r^{2}}+\frac{T}{M} \sin \beta,
\end{aligned}
$$

where $T$ is the thrust acting on the spacecraft, $M$ is the instantaneous mass of the spacecraft and $\beta$ is the control angle measured clockwise (figure 1) from the local horizontal to the thrust direction.

\subsection{Optimal control problem}

In order to obtain the control variable profile, Lagrange multipliers, also known as co-state variables are introduced. Let $p_{r}, p_{\varphi}, p_{u}, p_{v}$ be the co-state variables. Following the principles of Pontryagin, the Hamiltonian is formed as

$$
H=p_{r} \dot{r}+p_{\phi} \dot{\phi}+p_{u} \dot{u}+p_{v} \dot{v}
$$

and the equations governing the variations in the co-state variables are given by

$$
\begin{array}{ll}
\dot{p}_{r}=-\frac{\partial H}{\partial r}, & \dot{p}_{\phi}=-\frac{\partial H}{\partial \phi}, \\
\dot{p}_{u}=-\frac{\partial H}{\partial u}, & \dot{p}_{v}=-\frac{\partial H}{\partial v},
\end{array}
$$

and derived as

$$
\begin{aligned}
& \dot{p}_{r}=\frac{p_{\phi} u}{r^{2}}+p_{v}\left[\frac{u^{2}}{r^{2}}\right]-\frac{p_{u} u v}{r^{2}}, \\
& \dot{p}_{\phi}=0, \\
& \dot{p}_{u}=-\frac{p_{\phi}}{r}-\frac{2 p_{v} u}{r}+\frac{p_{u} v}{r},
\end{aligned}
$$




$$
\dot{p}_{v}=-p_{r}+\frac{p_{u} u}{r}
$$

The optimal control angle profile is obtained by minimizing the Hamiltonian at each instant of time with respect to the control variable. Because the control variable is unbounded in this case, the Hamiltonian is minimum when

$$
\frac{\partial H}{\partial \beta}=0
$$

and the control variable is given by

$$
\beta=\tan ^{-1}\left[\frac{p_{v}}{p_{u}}\right] .
$$

Also, it can easily be verified that $\left(\partial^{2} H / \partial \beta^{2}\right)>0$ and this condition and equation (11) are the necessary and sufficient conditions for the Hamiltonian to be minimum and the control to be optimum.

\subsection{Two-Point Boundary Value (TPBV) problem}

To obtain the optimal profile for the thrust direction, the equations for the co-state variables are to be solved, in addition to the governing equations of motion. Though the initial values of the state variables at the initial time $\left(t_{i}\right)$ are known, the initial values for the co-state variables are unknown and they must be chosen appropriately. Also, the terminal conditions on the state variables are specified except on the range angle. Since the time is to be minimized and the range angle is related to time, it is left as a free variable. The Pontryagin's principle requires that the terminal value of a costate variable corresponding to a free state variable is to be zero at the final instant of time $\left(t_{f}\right)$. So, $p_{\phi}\left(t_{f}\right)=0$. The other co-state variables are free at the final instant of time $\left(t_{f}\right)$ since the terminal conditions of corresponding state variables are fixed. Because of the equation (8), $p_{\phi}\left(t_{i}\right)=0$.

The optimal control problem which is converted into a two-point boundary value problem is stated as follows:

The differential equations (1)-(4) and (7)-(10) are to be solved with the following boundary conditions,

$$
\begin{gathered}
r\left(t_{i}\right)=r_{i}, \varphi\left(t_{i}\right)=\varphi_{i}, u\left(t_{i}\right)=u_{i}, v\left(t_{i}\right)=v_{i}, \\
p_{r}\left(t_{i}\right)=?, p_{\varphi}\left(t_{i}\right)=0, p_{v}\left(t_{i}\right)=?, p_{u}\left(t_{i}\right)=?
\end{gathered}
$$

$r\left(t_{f}\right)=r_{f}, \varphi\left(t_{f}\right)=$ free, $u\left(t_{f}\right)=u_{f}, v\left(t_{f}\right)=v_{f}$, $p_{r}\left(t_{f}\right)=$ free, $p_{\varphi}\left(t_{f}\right)=0, p_{v}\left(t_{f}\right)=$ free, $p_{u}\left(t_{f}\right)=$ free while the optimal thrust direction is described by equation (12).

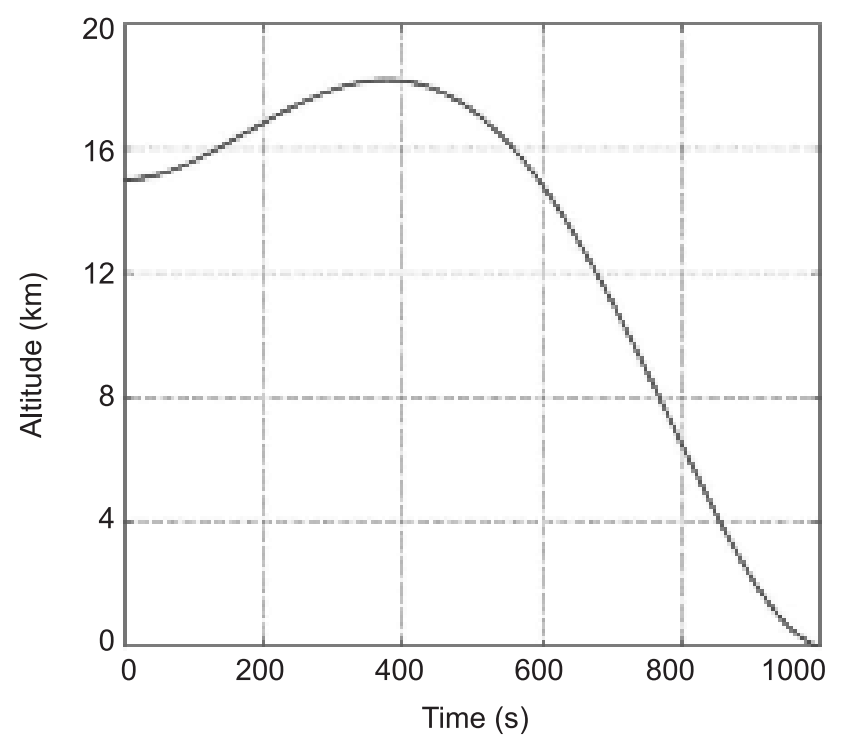

Figure 2. Profile of the altitude for an optimal landing trajectory.

Since the Hamiltonian is homogeneous in costate variables, without loss of generality, one of the co-state variables can be fixed at a value at the initial time, which results in scaling of the other two co-state variables.

\subsection{Solution of Two-Point Boundary Value (TPBV) problem}

The solution of the problem is now obtained by appropriate values for the co-state variables at the initial time. This is achieved by an optimization technique such as controlled random search. A version of this search technique as presented in is used. The functioning of this technique is briefly described in the following section. The terminal boundary conditions to be achieved are handled through a function that is set as the objective function in these techniques. The objective function to meet the terminal conditions at the final instant of time is formulated as

$$
F=\left(u\left(t_{f}\right)-u_{f}\right)^{2}+\left(v\left(t_{f}\right)-v_{f}\right)^{2} .
$$

The function $F$ must be zero if the terminal conditions are to be met. Also, for the appropriate choice of the co-state variables, bounds in which they are likely to vary are to be given. For each set of initial co-state values, the differential equations (1)-(4) and (7)-(10) are numerically integrated with the optimal thrust direction described by equation (12). The numerical integration is terminated when the instantaneous radial distance $(r)$ equals the desired radial distance and the function value is evaluated. 


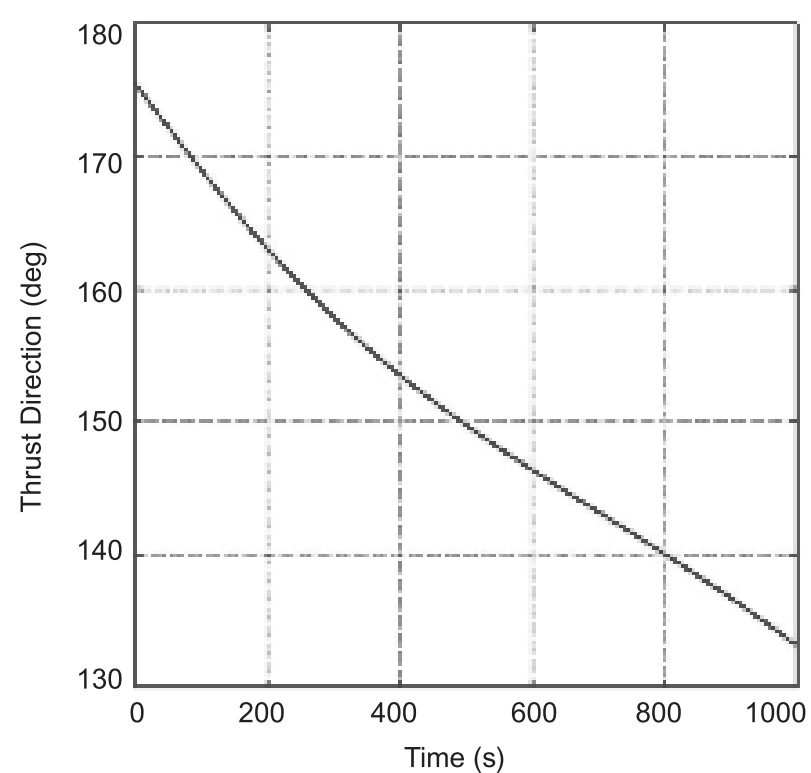

Figure 3. Optimal thrust direction profile.
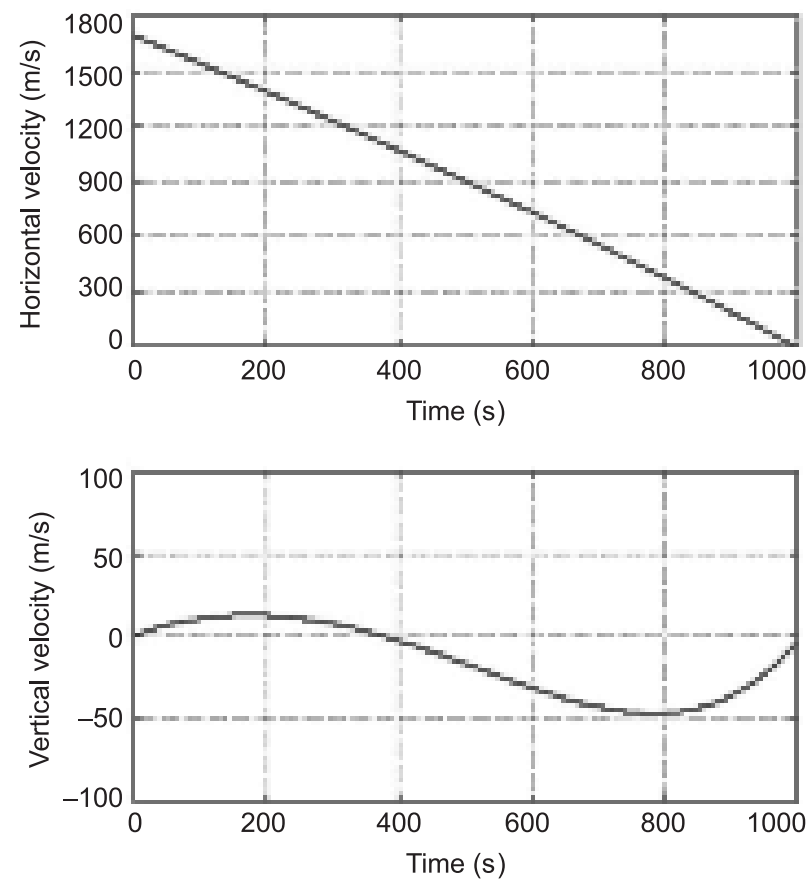

Figure 4. Components of velocity during powered braking.

\subsection{Controlled Random Search (CRS): Global optimization technique}

The CRS algorithm is an effective tool for global optimization. It works even when the differentiability requirements cannot be ensured in the feasible domain $(V)$. For initiating the algorithm, no initial guess value, except for an estimate of $V$, is needed. It works in two phases. In the first phase, a set of $N$ (suggested value is $10 n+10, n$ being the number of variables involved) random feasible

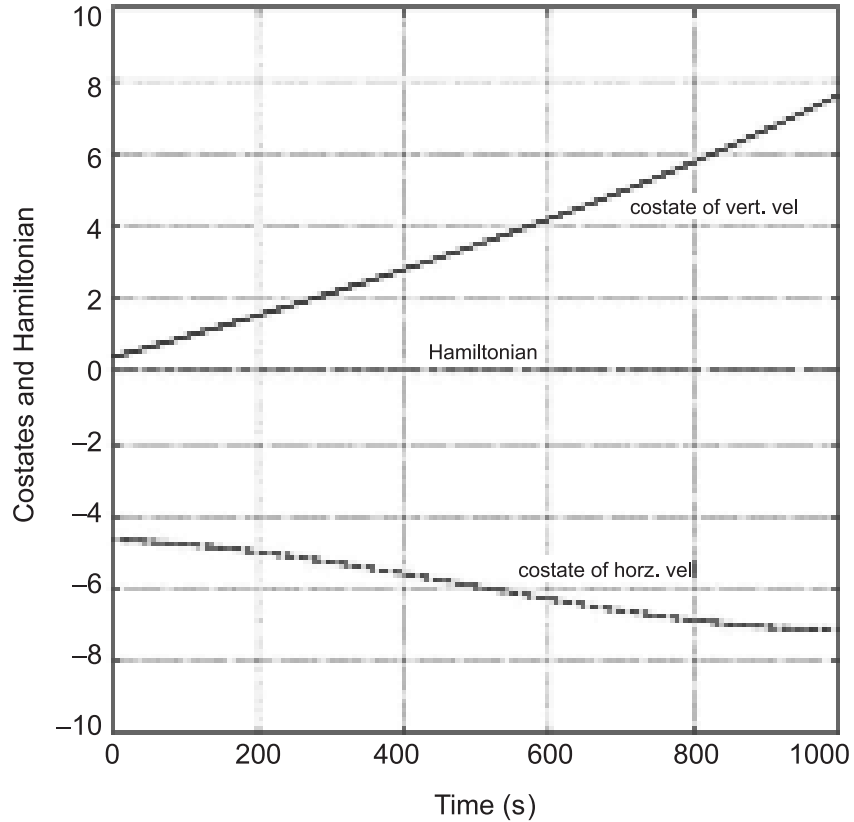

Figure 5. Co-state variables and Hamiltonian for optimal trajectory.

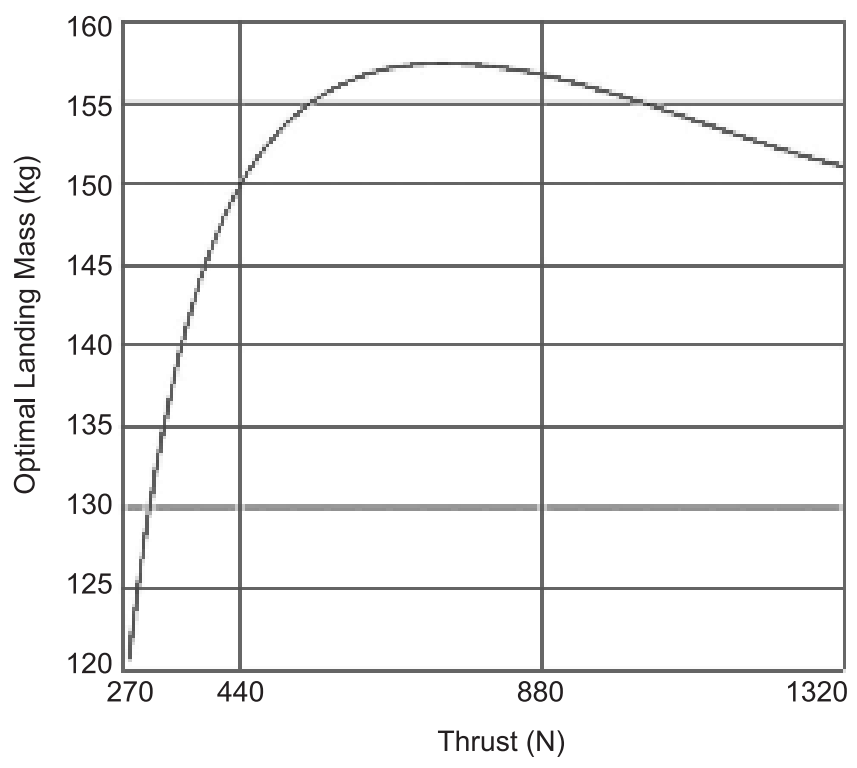

Figure 6. Optimal landing mass for different thrust levels.

points are generated from $V$, and $F$ is evaluated at each of these points and the information is stored as matrix $A$ of order $(N, n+1)$. The maximum and minimum values $F_{M}, F_{L}$ of $F$ and the corresponding points $M$ and $L$ are then identified. In the second phase, these random points are manipulated iteratively to yield a better candidate for global solution. To this extent, at each iteration $n$ arbitrary distinct points are chosen from $A$. A new point $T=2 G-L, G$ being the centroid of these points, is generated and if $T$ is in $V$, then $F_{T}$ is evaluated. If $F_{T}<F_{M}$, then $F_{M}$ and $M$ in $A$ are 
Table 1. Lunar parking orbit sizes and final landing masses. Strategy: Parking orbit $\rightarrow$ Intermediate orbit $\rightarrow$ Powered landing phase.

\begin{tabular}{cccc}
\hline $\begin{array}{c}\text { Size of the lunar } \\
\text { parking orbit }(\mathrm{km})\end{array}$ & $\begin{array}{c}\text { Duration of } \\
\text { powered } \\
\text { phase }(\mathrm{s})\end{array}$ & $\begin{array}{c}\text { Landing } \\
\text { mass }(\mathrm{kg})\end{array}$ & $\begin{array}{c}\text { Angle of longitudinal } \\
\text { axis from local } \\
\text { horizontal }(\mathrm{deg})^{*}\end{array}$ \\
\hline $100 \times 100$ & 1036.99 & 149.766 & 124.638 \\
$100 \times 50$ & 1012.06 & 152.126 & 129.336 \\
$100 \times 25$ & 1001.14 & 153.144 & 132.041 \\
$100 \times 20$ & 999.128 & 153.323 & 132.607 \\
$100 \times 15$ & 997.146 & 153.498 & 133.185 \\
$100 \times 10$ & 995.210 & 153.671 & 133.773 \\
$100 \times 5$ & 993.340 & 153.845 & 134.372 \\
\hline
\end{tabular}

${ }^{*}$ Required angle is $90^{\circ}$.

Table 2. Altitudes for the end of horizontal braking and the final masses. (Intermediate orbit: $100 \times 15 \mathrm{~km}$; Horz. Vel: $0 \mathrm{~m} / \mathrm{s}$; Vert. Vel: $50 \mathrm{~m} / \mathrm{s}$ ).

\begin{tabular}{cccc}
\hline & & \multicolumn{2}{c}{ At the end of horizontal braking } \\
\cline { 3 - 4 } $\begin{array}{c}\text { Target } \\
\text { altitude } \\
(\mathrm{km})\end{array}$ & $\begin{array}{c}\text { Duration of } \\
\text { horz. braking } \\
(\mathrm{s})\end{array}$ & Mass $(\mathrm{kg})$ & $\begin{array}{c}\text { Angle of longitudinal } \\
\text { axis from local } \\
\text { horizontal }(\mathrm{deg})\end{array}$ \\
\hline 1.0 & 976.48 & 156.757 & 136.972 \\
2.0 & 975.75 & 156.864 & 137.147 \\
3.0 & 975.01 & 156.970 & 137.284 \\
4.0 & 974.28 & 157.076 & 137.461 \\
5.0 & 973.56 & 157.181 & 137.638 \\
\hline
\end{tabular}

Table 3. Optimal vertical velocities.

\begin{tabular}{ccccccr}
\hline & & & & & & \multicolumn{2}{c}{$\begin{array}{c}\text { At the end of vertical } \\
\text { descent phase }\end{array}$} \\
\cline { 1 - 2 } $\begin{array}{c}\text { Altitude } \\
(\mathrm{km})\end{array}$ & $\begin{array}{c}\text { Vertical } \\
\text { velocity } \\
(\mathrm{m} / \mathrm{s})\end{array}$ & Mass $(\mathrm{kg})$ & Time $(\mathrm{s})$ & & $\begin{array}{c}\text { Landing } \\
\text { mass }(\mathrm{kg})\end{array}$ & Time $(\mathrm{s})$ \\
\hline 3.0 & 61.05 & 157.406 & 970.514 & & 150.271 & 49.107 \\
4.0 & 70.55 & 157.985 & 966.065 & & 149.764 & 56.669 \\
\hline
\end{tabular}

replaced by $F_{T}$ and $T$. Otherwise, $T$ is discarded and a new $T$ is generated. Treating any replacement as a success and setting the minimum success rate as 0.5 , the efficiency of the procedure is enhanced by making use of the secondary trial point $Q=(3 G+L) / 4$. If $T$ or $Q$ is a success, a third trial is also made with $X=2.5$ ( $T$ or $Q$ ) $-1.5 L$ and the best (i.e., with the least $F$ value) of Tor $Q$ or $X$ is used for replacement. The iteration continues till $F_{L}$ falls below the prefixed threshold value.

\section{Results and discussion}

The functioning of the formulation is demonstrated assuming $300 \mathrm{~kg}$ of mass in $100 \mathrm{~km}$ circular lunar parking orbit. The other parameters used are: $440 \mathrm{~N}$ for the thruster and $310 \mathrm{~s}$ for specific impulse.
The powered braking during which optimal control is exercised starts from the periapsis of $100 \times 15 \mathrm{~km}$ orbit. Terminal vertical velocity of $5 \mathrm{~m} / \mathrm{s}$ and a horizontal velocity of $0 \mathrm{~m} / \mathrm{s}$ are the conditions achieved at touchdown. The altitude profile of the optimal trajectory is plotted in figure 2. Initially the altitude of the probe rises to maximize horizontal braking that minimizes the gravity loss. The profile of the thrust direction is plotted in figure 3 . The near linear variation of the profile can be noted. The horizontal and vertical velocities are depicted in figure 4 . The co-states and Hamiltonian are plotted in figure 5. Clearly, the Hamiltonian is invariant as required by Pontryagin's maximum principle on the optimal trajectory.

Because a landing mission requires vertical landing, apart from near-zero velocities at the time of touchdown, different strategies are explored to identify an appropriate one. 
The following strategies are studied for soft landing on the Moon:

- by initiating the powered braking from the lunar parking orbit itself and directly landing,

- by initiating powered braking from an intermediate orbit and directly landing,

- by splitting the powered braking into two phases: (i) powered horizontal braking phase that ends at a low altitude with a small vertical velocity and a zero horizontal velocity (ii) a vertical descent phase ensuring the required touchdown velocity.

The merits and demerits of the strategies are discussed.

\subsection{Strategy: Parking orbit $\rightarrow$ powered landing phase}

A direct landing with a target vertical velocity of $5 \mathrm{~m} / \mathrm{s}$ at touchdown is attempted from a $100 \times 100 \mathrm{~km}$ orbit. In this case, the powered braking starts from an altitude of $100 \mathrm{~km}$. The optimal landing masses for different thrust levels are plotted in figure 2. The thrust level that yields a maximum optimal landing mass is $700 \mathrm{~N}$. However, the thrust level of presently available Indian engine being $440 \mathrm{~N}$, the options available are restricted to $440 \mathrm{~N}$ and $880 \mathrm{~N}$ (two engines) thrusters. Though the landing mass is about $7 \mathrm{~kg}$ more for $880 \mathrm{~N}$ thrusters, the dry mass requirements of the second engine being about $10 \mathrm{~kg}$, the useful landing mass becomes less. So, in this case single $440 \mathrm{~N}$ thruster gives the optimal useful landing mass of $149.766 \mathrm{~kg}$ and the propellant consumption is $150.234 \mathrm{~kg}$. However, the orientation of the longitudinal axis (thrust direction) of the probe from the local horizontal at touchdown is $124.638 \mathrm{deg}$ (table 1 ) implying that vertical touchdown is not possible with this strategy.

\subsection{Strategy: Parking orbit $\rightarrow$ intermediate orbit $\rightarrow$ powered landing phase}

The use of intermediate orbits in increasing the optimal landing mass is discussed in this section. Various intermediate orbit sizes are considered with a $440 \mathrm{~N}$ thruster and a touchdown velocity of $5 \mathrm{~m} / \mathrm{s}$. Powered braking starts from the periapsis of the intermediate orbit. Table 1 presents the final landing masses for these orbit sizes. It is clear that the strategy of initiating the horizontal braking from an intermediate orbit is advantageous. This provides about $4 \mathrm{~kg}$ of additional optimal landing mass compared to the strategy of initiating the braking from the parking orbit itself. Also, only a marginal increase is obtained by reducing the periapsis of the intermediate orbit. So, the intermediate orbit size is chosen depending on the actual mission navigation accuracy and safety requirements. For further studies an intermediate orbit size of $100 \times 15 \mathrm{~km}$ is considered. The mass at this orbit is $298.087 \mathrm{~kg}$ after $1.92 \mathrm{~kg}$ of fuel is spent for orbit reduction from $100 \times 100 \mathrm{~km}$ orbit. The orientation of the longitudinal axis (thrust direction) of the probe from the local horizontal at touchdown is also given in table 1 . They are much different from the required ones for the vertical landing. For vertical landing, the longitudinal axis that is perpendicular to the plane of the landing gear must be along the local vertical.

\subsection{Strategy: Parking orbit $\rightarrow$ intermediate orbit $\rightarrow$ powered horizontal braking $\rightarrow$ landing by powered vertical phase}

The above two strategies do not satisfy the requirement for vertical landing. So, the powered braking is executed in two phases:

- powered horizontal braking phase

- vertical landing phase.

Powered horizontal braking phase is initiated from the periapsis of an intermediate orbit and is terminated at a low altitude above the surface of the Moon. In this phase, the horizontal component of the velocity is neutralized and a small target vertical velocity is achieved. This target vertical velocity is also neutralized during the powered vertical phase. Since vertical landing phase requires a constant attitude of $90^{\circ}$, appropriate target vertical velocity is chosen using the formulation that ensures constant orientation. At the end of horizontal powered braking, the probe's attitude makes an angle of $50^{\circ}$ with respect to local vertical. Required attitude correction is carried out before the start of the vertical landing. The altitude and the vertical velocity at the end of horizontal braking must be appropriately chosen to meet the mission accuracy and safety requirements.

\subsubsection{Target altitude at the end of horizontal braking}

For various target altitudes at which the zero horizontal velocity is achieved and horizontal braking is terminated, the optimal masses are given in table 2 . Also the thrust direction angle at the end of horizontal braking is given. The target vertical velocity that is to be achieved at the end of horizontal braking is taken as $50 \mathrm{~m} / \mathrm{s}$ for this analysis. The variation in the optimal masses at the end of horizontal braking is only marginal and also the attitude correction requirements remain almost the same for 
all the cases. So, the sensitivity of the target conditions at the end of horizontal braking to the error sources like bias in accelerometer and gyros, uncertainties in periapsis altitude, etc., should be studied to fix a target altitude. The target altitude must be chosen to accommodate the expected errors in altitude and velocity. A terminal altitude of $3 \mathrm{~km}$ and $4 \mathrm{~km}$ is considered for further analysis.

\subsubsection{Target velocity at the end of horizontal braking}

The vertical velocity at the end of horizontal powered braking must be chosen such that the attitude correction requirements do not exist during vertical descent. Table 3 presents the optimal vertical velocities for the target altitudes 3 and $4 \mathrm{~km}$. These are obtained by generating optimal trajectories in both the phases in an iterative manner. An altitude of $2 \mathrm{~m}$ and a velocity of $0 \mathrm{~m} / \mathrm{s}$ are set as the targets to be achieved at the end of the vertical landing phase. After this, the probe has a free fall ensuring a touchdown velocity of about $5 \mathrm{~m} / \mathrm{s}$. If $3 \mathrm{~km}$ is the choice for the end of horizontal braking, a target velocity of $61.05 \mathrm{~m} / \mathrm{s}$ is to be achieved to enable a vertical descent with no attitude manoeuver. That is, any other target velocity will involve attitude orientation manoeuvers during vertical descent. Similarly, a $4 \mathrm{~km}$ target altitude requires a target vertical velocity of $70.55 \mathrm{~m} / \mathrm{s}$ at the end of horizontal braking. The landing mass in these cases differs by $0.5 \mathrm{~kg}$. However, the time required for the thrust direction angle correction for the start of vertical descent and the velocity added to the probe during this time is not accounted in this study. But this can be easily incorporated by choosing conditions at the end of horizontal braking appropriately.

This strategy yields an optimal lander mass of $150.271 \mathrm{~kg}$ compared to $153.498 \mathrm{~kg}$ of the second strategy. Though the optimal lander mass is $3.2 \mathrm{~kg}$ less, the vertical soft landing requires adaptation of this strategy.

\section{Conclusions}

The optimal control problem of soft landing a probe from a lunar parking orbit is solved. This formulation is used to analyse various strategies for vertical soft landing and to fix some of the design parameters:

- thrust level,

- intermediate orbit size,

- altitude at the end of horizontal braking phase and

- velocity at the end of horizontal braking that involves no control effort during the vertical descent phase.

These design parameters ensure maximum landing mass. The strategy: parking orbit $\rightarrow$ intermediate orbit $\rightarrow$ powered horizontal braking $\rightarrow$ landing by powered vertical phase, yields the maximum optimal landing mass satisfying landing requirements. For other probe masses a similar analysis can be carried out with the recommended strategy and the design parameters can be chosen.

\section{References}

Kawakatsu Y, Kaneko Y and Takizawa Y 1998 Trajectory design of SELENE Lunar orbiting and Landing; AAS 98-320, AAS/AIAA Astrodynamics Conference.

Kirk D E 1970 'Optimal Control Theory: An Introduction', Prentice Hall.

Oono H, Ishikawa S, Nakajima K, Hayashi K and Odaka R 1998 Navigation and Guidance Error Analysis of Lunar Lander Considering Orbit Determination Error; AAS 98-319, AAS Astrodynamics Conference.

Price W L 1983 Global optimization by controlled random search; J. Optimization Theory and Applications $\mathbf{4 0}$ 333-348.

Subba Rao P V and Ramanan R V 1992 Optimum Rendezvous Transfer Between Coplanar Heliocentric Elliptic Orbits Using Solar Sail; AIAA Journal of Guidance, Control and Dynamics 15(6) 1507-1509.

Vasile M and Flobergghagen R 1998 Optimal Trajectories For Lunar Landing Missions; AAS 98-321, AAS Astrodynamics Conference. 